\title{
Catheter-based infrared light scanner as a tool to assess conduit quality in coronary artery bypass surgery
}

Nicholas Burris, BS, ${ }^{\text {a }}$ Kimberly Schwartz, RNFA, MBA, a Cha-Min Tang, MD, PhD, ${ }^{\text {b }}$ M. Samir Jafri, PhD, b,c Joseph Schmitt, PhD, ${ }^{d}$ Michael H. Kwon, BS, ${ }^{a}$ Ozeki Toshinaga, PhD, ${ }^{a}$ Junyan Gu, MD, PhD, ${ }^{a}$ Jamie Brown, MD, ${ }^{a}$ Emile Brown, BS, ${ }^{a}$ Richard Pierson III, MD, ${ }^{a}$ and Robert Poston, $\mathrm{MD}^{\mathrm{a}}$

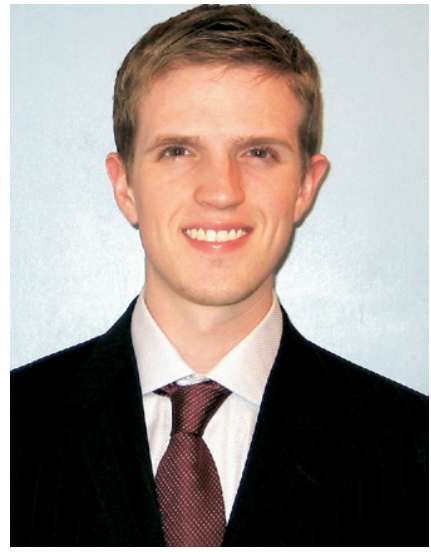

Dr Burris
Objectives: Endothelial disruption within saphenous vein and radial artery grafts increases thrombosis risk. However, no clinically applicable method for imaging the intima currently exists. We used a novel infrared imaging technology, optical coherence tomography (OCT; LightLab Imaging, Inc, Westford, Mass), to visualize the intima within harvested conduits.

Methods: Conduits were procured endoscopically (37 saphenous vein grafts and 8 radial artery grafts) or with the open technique ( 9 radial artery grafts) from 50 patients. Surplus segments were analyzed by means of OCT for evidence of preexisting pathology or traumatic injury. Focal plaques in radial artery grafts and the intimal hyperplasia area in saphenous vein grafts were quantified as having an intimal/medial thickness ratio of greater than 0.5. Biopsy specimens were obtained for histologic confirmation and to analyze matrix metalloproteinase 2 levels (saphenous vein grafts) and prostacyclin/nitric oxide metabolites (radial artery grafts). Interobserver $\kappa$ coefficients and a Bland-Altman analysis were used to determine the reproducibility and accuracy of OCT interpretations.

Results: Radial artery imaging revealed plaque in $76 \%$. Endoscopically harvested vessels showed intraluminal clot (38\%) and intimal tears ranging from severe (6\%) to mild $(88 \%)$. In saphenous vein grafts intimal thickening was detected in $86 \%$ and intraluminal clotting in $68 \%$. The intimal/medial thickness ratio determined by means of OCT correlated directly with matrix metalloproteinase 2 levels $(R=$ $0.6804)$ in saphenous vein grafts and inversely with metabolites of prostacyclin $(R=-0.55)$ and nitric oxide $(R=-0.58)$ in radial artery grafts. OCT imaging was reproducible (interobserver $\kappa$ coefficients of $>0.81$ for the characterization of plaque types) and showed a strong correlation with histology $(R=0.8, P<.001)$.

Conclusions: OCT imaging provides an accurate, real-time, and reproducible means for assessing saphenous vein graft and radial artery graft bypass conduits. As a quality assurance tool, this technology might afford a more objective basis for conduit selection.

Received for publication May 1, 2006; revisions received Aug 13, 2006; accepted for publication Sept 7, 2006.

Address for reprints: Robert Poston, MD, Division of Cardiac Surgery, University of Maryland School of Medicine, N4W94 22 S Greene St, Baltimore, MD 21201 (E-mail: rposton@smail.umaryland.edu).

Joseph Schmitt is the Chief Technical Officer of LightLab Imaging, Inc, manufacturer of the OCT probe.

J Thorac Cardiovasc Surg 2007;133:419-27

0022-5223/\$32.00

Copyright (C) 2007 by The American Association for Thoracic Surgery

doi:10.1016/j.jtcvs.2006.09.056
$\mathrm{T}$ hrombosis of saphenous vein grafts (SVGs) develops in 5\% to $10 \%$ in the first week and $20 \%$ to $30 \%$ over the first year after coronary artery bypass grafting $(\mathrm{CABG})$. This problem predicts the need for reoperation ${ }^{1}$ and accounts for a substantial proportion of the well-known limitations of long-term patency associated with this conduit. ${ }^{2}$ Because easily assessable end points, such as perioperative symptoms, electrocardiographic changes, and myocardial enzyme release are poor surrogates, almost all of these events go undetected. Given that more than 400,000 patients are treated with CABG annually, acute graft loss remains one of the most underdiagnosed and underreported problems in medicine. ${ }^{3,4}$ 


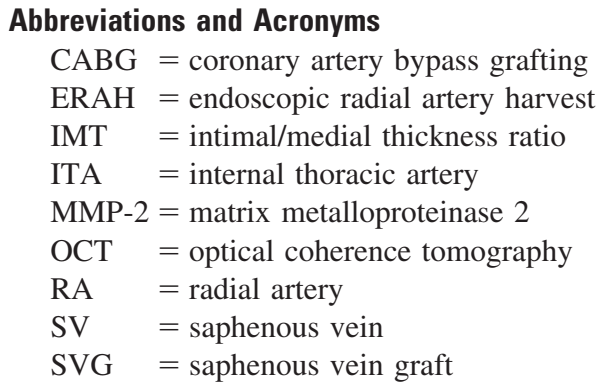

Imperfect anastomotic technique receives the majority of the blame for early graft failures. The role of intimal quality of the conduit has not been investigated in as much detail and might be underappreciated. The stark contrast in the rate of early failure between the saphenous vein (SV) and internal thoracic artery (ITA) strongly implicates characteristics unique to the conduit, rather than anastomotic technique or graft outflow, as a primary cause of failure. One of the major issues is relative exposure to traumatic injury during procurement of each conduit. Although the ITA is harvested with a "no-touch" technique, saphenous vein and radial artery (RA) harvest frequently involve manual distension to resolve spasm and identify branches, followed by hypoxic storage. ${ }^{5,6}$ These factors disrupt intimal integrity and increase the risk of early failure. ${ }^{7,8}$

Although the ITA is the first-choice conduit, SV and RA grafts continue to be used for one or more grafts, even where bilateral ITA grafting is done. The lack of a convenient means to objectively assess intimal quality in real time has made it difficult to establish the effect of conduit selection practices. Catheter-based optical coherence tomography (OCT) has been shown to provide vascular images that yield morphologic information about the tissue that approaches histologic resolution. ${ }^{9,10}$ The purpose of this study was to determine the feasibility of applying OCT toward the screening of conduits for CABG.

\section{Methods}

\section{Patient Enrollment and Data Management}

After institutional review board approval (UMB protocol no. $\mathrm{H} 25350$ ), all subjects provided informed consent before enrollment. A total of 695 patients underwent isolated off-pump coronary artery bypass at our institution from November 2002 until March 2006, and 245 patients were enrolled. An unselected subset of $52(10.5 \%)$ of these patients had an OCT evaluation performed on surplus segments of 37 SVGs, 17 RA grafts, and 3 ITA grafts. Demographics, preoperative risk factors and medications, and intraoperative and postoperative data were prospectively imported into a relational database (Table 1).

\section{Surgical Technique}

Four surgeons experienced in off-pump coronary artery bypass enrolled patients. After median sternotomy, the left ITA was
TABLE 1. Patient characteristics

\begin{tabular}{lccc}
\hline Characteristic & $\begin{array}{c}\text { EVH } \\
(\mathbf{n}=\mathbf{3 7})\end{array}$ & $\begin{array}{c}\text { Open RA } \\
(\mathbf{n}=\mathbf{9})\end{array}$ & $\begin{array}{c}\text { ERAH } \\
(\mathbf{n = 8}=\end{array}$ \\
\hline Age (y) & $64.1 \pm 9.5$ & $62.3 \pm 7.8$ & $61.6 \pm 3.6$ \\
Male sex & $68 \%(25 / 37)$ & $78 \%(7 / 9)$ & $63 \%(5 / 8)$ \\
Hypertension & $73 \%(27 / 37)$ & $55 \%(5 / 9)$ & $75 \%(6 / 8)$ \\
Smoking history & $46 \%(17 / 37)$ & $78 \%(7 / 9)$ & $63 \%(5 / 8)$ \\
Hypercholesterolemia & $84 \%(31 / 37)$ & $88 \%(8 / 9)$ & $78 \%(6 / 8)$ \\
PVD & $11 \%(4 / 37)$ & $22 \%(2 / 9)$ & $13 \%(1 / 8)$ \\
Renal failure & $5 \%(2 / 37)$ & $0 \%(0 / 9)$ & $13 \%(1 / 8)$ \\
Diabetes & $46 \%(17 / 37)$ & $33 \%(3 / 9)$ & $38 \%(3 / 8)$ \\
\hline
\end{tabular}

$E V H$, Endoscopic vein harvest; $R A$, radial artery; $E R A H$, endoscopic radial artery harvest; $P V D$, peripheral vascular disease.

harvested in all patients. Eight of the 52 enrolled patients underwent endoscopic RA harvest (ERAH), whereas all SVGs were harvested endoscopically (VasoView6; Guidant Systems, Inc, Minneapolis, Minn). Heparin was given at the completion of the ITA takedown (but not before initiating endoscopic conduit harvest) at a dose calculated to obtain an activated clotting time of greater than 300 seconds and heparin of greater than $2 \mathrm{IU} / \mathrm{mL}$ according to protamine titration (HMS heparin assay cartridges; Medtronic, Inc, Minneapolis, Minn). Preoperative aspirin (325 mg by mouth daily) was continued and given within 6 hours after surgical intervention. Procured SVGs and RA grafts were initially flushed with heparinized saline by using syringe injection, with no methods used to control the distending pressure (54\%), to prepare the conduits for grafting. The remainder of the conduits were flushed in a plasmalyte solution containing glyceryl trinitrate and verapamil ${ }^{11}$ using controlled distending pressure at less than 100 $\mathrm{mm} \mathrm{Hg}$. The proximal aortic anastomoses were performed first for each SVG or RA graft with a partial occluding aortic clamp.

\section{Ex Vivo OCT Analysis}

Discarded segments of saphenous veins or RAs from the distal end of the conduit were stored in Hank's balanced salt solution at $4^{\circ} \mathrm{C}$. Ex vivo evaluations by using OCT (LightLab Imaging, Inc, Westford, Mass) were completed within 2 hours of removal from the operative field. For the examination, a cannula (DLP Vessel Cannula; Medtronic, Inc) was inserted into one end of the segment, and the other end was occluded with a spring-loaded vascular clip. The OCT probe was introduced into the vessel though a Yconnector attached to the cannula, allowing for gentle infusion of Hank's balanced salt solution during imaging, and automated pullback images were obtained. Plaques visualized in OCT crosssectional images were categorized as fibrous, lipid laden, or calcified based on prior reported criteria for OCT. ${ }^{12}$ Intimal disease within the analyzed conduit was quantified by determining the maximum intimal/medial thickness ratio (IMT), and severity of calcification (none, mild, and severe) was quantified by means of techniques described in reports using intravascular ultrasonography. ${ }^{13}$ Harvesting injury was categorized as mild when intimal disruption was restricted to the ostium of branch points and severe when the tear affected the luminal surface. The percentage of ostium showing injury and the number of severe tears per centimeter were measured. 


\section{Histologic Examination}

Biopsy specimens for histologic processing were procured at the completion of the ex vivo scan. To exactly match the OCT images with the corresponding histopathologic sections, the vessel site at which the biopsy specimen was obtained was marked externally at the location of the catheter, visualized by the rotating infrared light at the catheter tip. These image-guided biopsy specimens were then stored in solution before being embedded and frozen in cutting compound (Tissue-Tek O.C.T., Redding, Calif). Additional sections were embedded in paraffin, sectioned at $5 \mu \mathrm{m}$, and stained with hematoxylin and eosin. Microscopic sections were analyzed for the percentage of luminal endothelial integrity by using the CD31 mAb (R\&D System, Inc), as previously described. ${ }^{14}$

\section{Enzyme-linked Immunosorbent Assays}

A portion of each RA vessel biopsy specimen was homogenized to analyze metabolites of nitric oxide $\left(\mathrm{NO}_{2} / \mathrm{NO}_{3}\right.$; Assay designs, Inc, Ann Arbor, Mich) and prostacyclin (6-keto-PGF1; Assay designs, Inc) by using colorimetric assays. Biopsy specimens of SVGs were analyzed with enzyme-linked immunosorbent assay kits for matrix metalloproteinase 2 (MMP-2) levels (Calbiochem, San Diego, Calif). Results were normalized against the dry weight of the homogenate sample and compared with OCT measurements of intimal and medial thickness. Clots retrieved from the lumen of the $\mathrm{SV}(\mathrm{n}=2)$ were analyzed for thrombin activity by means of incubation with the chromogenic substrate S-2238 (333 $\mu \mathrm{mol} / \mathrm{L}$, Chromogenix) in a reaction buffer for 30 minutes. The absorption of the reaction buffer was assessed at $405 \mathrm{~nm}$ and then compared with a standard curve to determine thrombin activity.

\section{Statistics}

The primary end point of this trial was to describe the prevalence of conduit abnormalities by using OCT and to validate the accuracy of OCT against a gold standard, histopathology. BlandAltman analysis was used to verify the degree of agreement of OCT with histopathology. Reproducibility was determined by defining the intraobserver and interobserver $\kappa$ correlation coefficients. Enzyme-linked immunosorbent assay data were matched with the corresponding OCT image analysis by determining the Pearson correlation coefficient. Statistical analysis was performed with the InStat statistical package with consultation of a biostatistician. The sponsors of the study had no role in the publication of these data.

\section{Results}

\section{Procurement-related Injury}

Normal RAs possess a 3-banded appearance on OCT scans that correspond to the internal elastic lamina of the intima, media, and adventitia (Figure 1, A). The strong contrast provided by the intima makes abnormalities, such as intimal tears and dissections, easily detectable. Intimal tears or dissections that involved the luminal surface of the RA were observed in $6 \%$ of the RA segments and were confirmed in each case by means of histologic examination. ERAH was associated with a unique pattern of intimal tears localized to the ostia of branch points in $88 \%$ of evaluated RAs (Figure 2). Of a total of 39 branches evaluated by means of OCT, $21 \%$ were observed to have intimal disruption at the branch
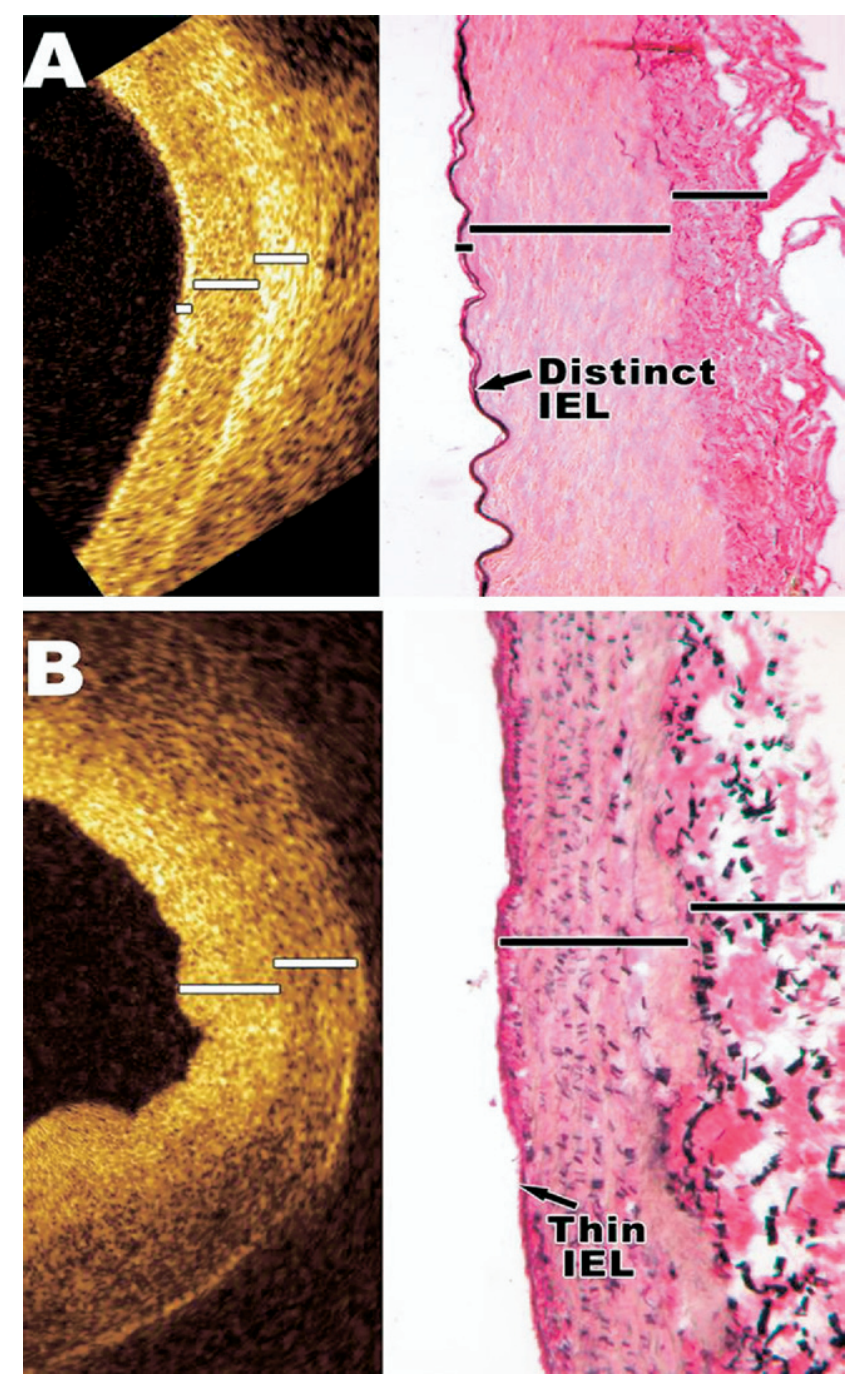

Figure 1. Normal appearance of the radial artery and saphenous vein on optical coherence tomographic scans. A, Radial arteries demonstrate a 3-banded appearance by optical coherence tomography corresponding to a bright thin intima, a dark thick media, and a bright diffuse adventitia. The internal elastic lamina (IEL) of the radial arteries is well developed, as demonstrated by Verhoff van Giesen staining (elastin) of a healthy radial artery sample. B, Healthy saphenous veins demonstrate a 2-banded appearance by means of optical coherence tomography corresponding to the media (inner layer) and adventitia (external layer). The intima of normal saphenous veins is not resolved because the IEL is less developed than in radial arteries, as demonstrated by elastin staining, limiting the ability of optical coherence tomography to resolve intimal disruption in the saphenous vein graft conduit.

ostia. RA segments that had been harvested with the open technique showed no severe intimal disruptions, and only $3 \%$ of branch ostia showed intimal injury $(P<.05$ for the incidence of ostial injury after ERAH vs open harvest, Fisher exact test). 

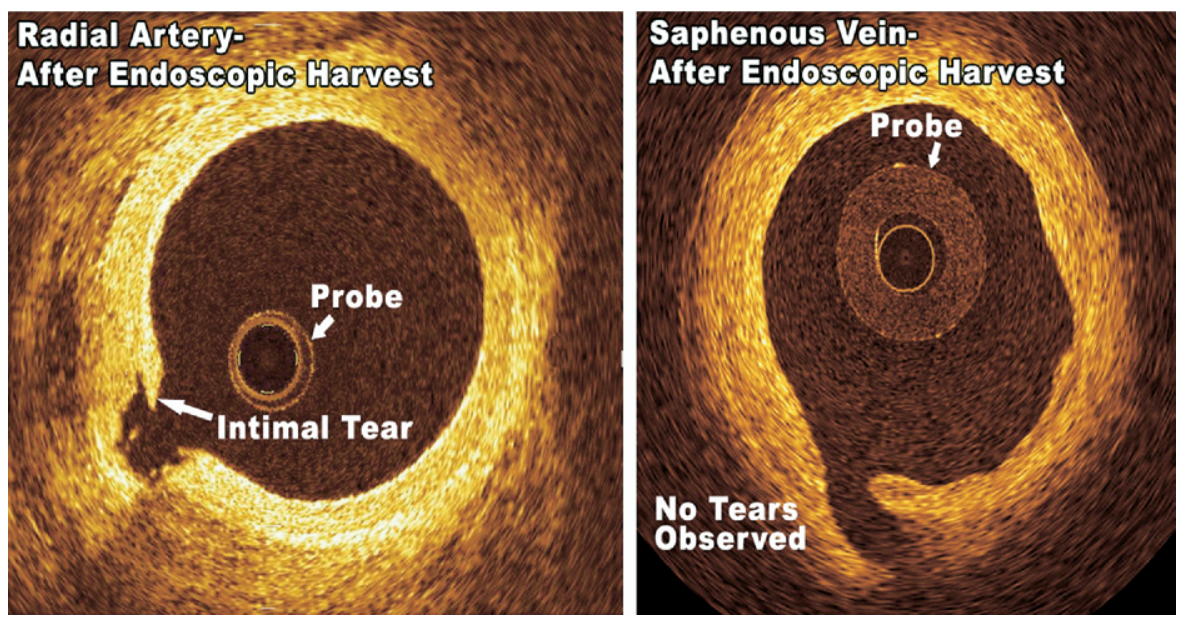

Figure 2. Optical coherence tomography provides real-time feedback on conduit injury in relation to harvesting technique. At least 1 intimal tear localized to the ostia of a branch point, such as that shown in this representative example, was observed in $\mathbf{8 8 \%}$ of imaged segments obtained after endoscopic radial artery harvest. Overall, $21 \%$ of branches showed this injury after endoscopic radial artery harvest, whereas none of the branches were injured after open radial artery harvest. In addition, saphenous vein grafts harvested by using endoscopic techniques did not show this pattern of intimal injury at branch point ostia, as illustrated in the example.

The less-developed internal elastic lamina of a normal saphenous vein provides a 2-banded image on OCT examination, without clear distinction of the intima (Figure 1, B). Severe intimal injury involving the lumen was detected in $2.7 \%$ of clinical SVG specimens and also confirmed by means of image-guided histology. Although endoscopic techniques were used for all SVGs, tears in the intima surrounding the ostia of SVG branches were not detected (Figure 2).

\section{Pre-existing Pathologic Condition}

Longitudinal reconstructions of OCT images illustrated that intimal thickening occurs as discrete eccentric abnormalities within the RA, which is consistent with an atherosclerotic plaque (Figure 3,A). Homogenously reflecting plaques without shadowing artifact were categorized as fibrous in $71 \%$ of evaluated RAs. Calcified plaque was characterized by the presence of a dark and well-delineated core within the bright thickened intima and was observed in $29 \%$ of
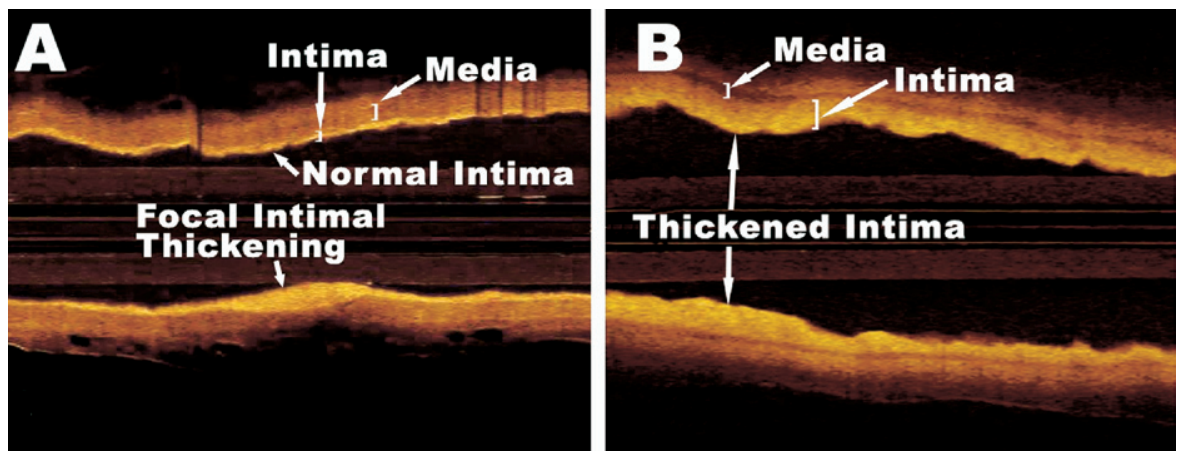

Figure 3. Focal plaque in radial arteries and diffuse intimal hyperplasia in saphenous veins. A, Intimal thickening in radial arteries was most commonly observed to be eccentric and focal. This representative longitudinal reconstruction of the data obtained from an optical coherence tomographic examination demonstrates a discrete area of intimal thickening immediately adjacent to areas with normal intimal thickness. These eccentric intimal thickenings were defined as plaque. B, In contrast to the focal intimal thickening of the radial artery, intimal disease in the saphenous vein graft was manifested as concentric and diffuse thickening consistent with intimal hyperplasia. This representative longitudinal reconstruction shows this process is occurring on both sides of the vein and throughout the length of the imaged segment. 

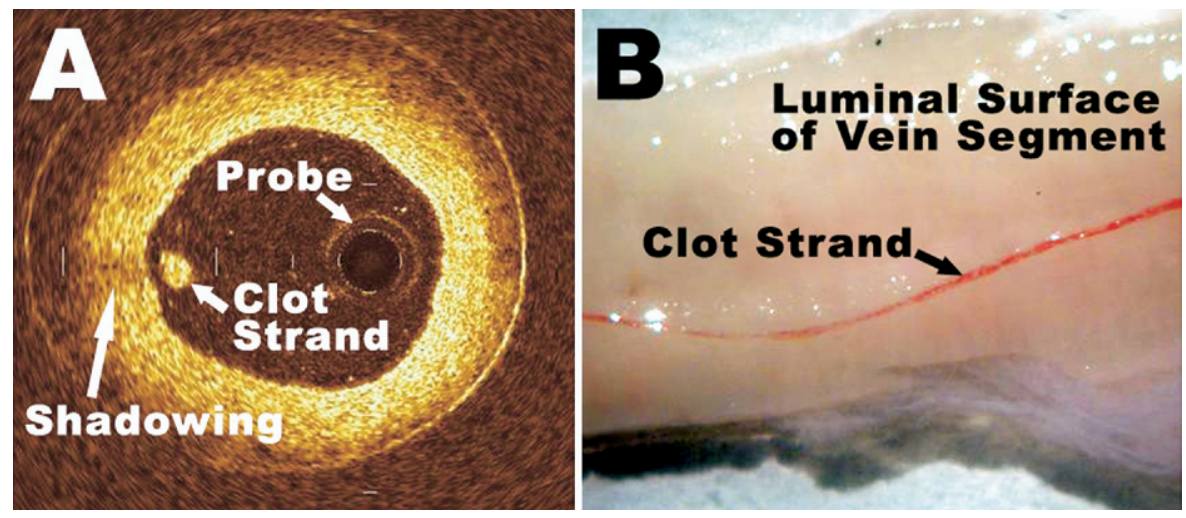

Figure 4. Clot strands observed in saphenous veins and radial arteries after endoscopic harvest. A, A luminal irregularity is observed on this representative optical coherence tomographic image that shows a characteristic shadowing artifact behind the strand. This shadowing is the result of erythrocytes within the clot strand that strongly attenuate the near-infrared light signal used by optical coherence tomography. B, Conduits with imaged clot strands were opened longitudinally to expose the luminal surface. The strand imaged by means of optical coherence tomography was confirmed to be a clot strand on the basis of gross examination of this representative segment.

evaluated RAs. Lipid-laden plaque was distinguished by a "shadowing" artifact and was observed in $35 \%$ of evaluated RAs. Severe circumferential calcification of the intima was observed in only $1(6 \%)$ of the evaluated RAs. Discarded segments of ITAs $(\mathrm{n}=3)$ scanned with OCT showed no atherosclerotic plaque, calcification, or intimal trauma.

Compared with the RA, SVG segments showed a more diffuse and concentric pattern of intimal thickening (Figure $3, B)$, with an average IMT ratio of $0.94 \pm 0.55$ and intimal thickness of $197 \pm 105 \mu \mathrm{m}$. Only 14\% of SVGs analyzed showed a normal intima and were characterized by an intimal/medial thickness ratio less than 0.5. In addition, medial calcification, discriminated by means of OCT from intimal calcification, was found in $41 \%$ of SVGs.

\section{Intraluminal Clots}

OCT identified clots (Figure $4, A$ ) in $38 \%$ of endoscopically harvested RA and $68 \%$ of SVG segments. No clot strands were observed in RAs harvested with the open technique. The diagnosis of clotting was confirmed by means of direct gross examination of the longitudinally opened vessel (Figure $4, B)$, by means of histologic confirmation, and by means of biochemical analysis of the clot $(\mathrm{n}=2)$, which confirmed thrombin activity of $210 \pm 178 \mathrm{mU} / \mathrm{mg}$ dry clot weight.

\section{Correlation of OCT Findings With Biochemical \\ End Points}

The levels of MMP-2 within SVG homogenates varied widely (214.19 $\pm 138.28 \mathrm{ng} / \mathrm{g}$ tissue) and strongly correlated with OCT determination of IMT ratio measured within ex vivo SVG segments $(R=0.6804, P<.001)$. Prostacyclin levels in RA homogenates showed an inverse correlation with the OCT measurements of IMT ratio determined in same-vessel sections $(R=-0.58)$. Similarly, $\mathrm{NO}_{2} / \mathrm{NO}_{3}$ levels showed an equally strong inverse correlation with IMT ratio $(R=-0.55)$.

\section{Reproducibility and Accuracy}

There were strong interobserver $\kappa$ correlation coefficients noted for the categorization of plaques that were fibrous (0.81), calcified (0.85), and lipid laden (0.82). The ability of OCT to resolve the pathologically thickened intima from the media provided by OCT was established by the strong correlation noted between the IMT ratio calculated by means of OCT versus histology $(R=0.88, P<.001$, Figure $5)$. In addition, a small average discrepancy in the IMT ratio $(-0.07 \pm 0.22)$ and consistent variation were seen across a Bland-Altman plot derived from measurements obtained by using the 2 methods.

\section{Discussion}

It is widely appreciated that endothelial disruption, slow blood flow, and the hypercoagulable state have a synergistic influence on the development of intravascular thrombosis. Modern ultrasonic flow measurements provide reliable data on graft flow. The surgical revision or replacement of grafts with flow that is established intraoperatively to be suboptimal can reduce the effect of technical defects on early graft attrition. However, the static rate of graft failure over the last 20 years suggests that conduit integrity, hypercoagula- 

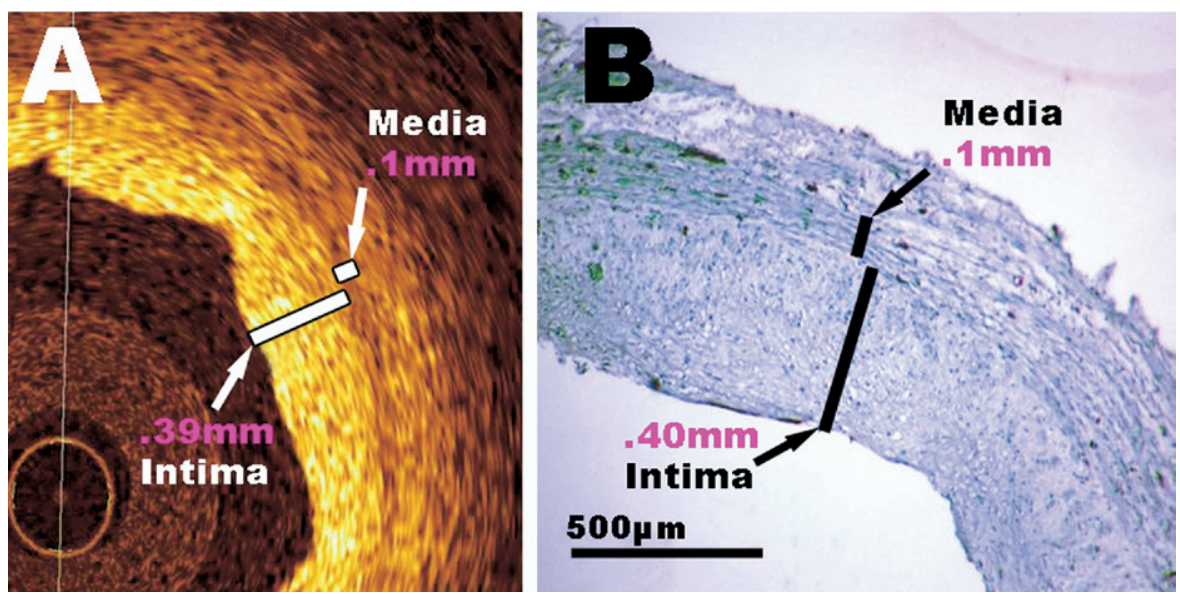

Figure 5. Accurate identification and measurement of intimal hyperplasia in saphenous veins. A, Optical coherence tomography has the ability to accurately identify the intima and media in saphenous veins with intimal hyperplasia (intima is thick enough to be resolved by means of optical coherence tomography). The intimal/medial thickness ratio was determined by using optical coherence tomography. B, Optical coherence tomographic measurements of intimal/medial thickness ratio were compared with histologic measurements (gold standard). Optical coherence tomographic measurements were found to strongly correlate with histologic measurements on analysis with a Bland-Altman plot.

bility, or both might be having an underappreciated effect on this problem. The integrity of the intimal layer has proved to be very difficult to quantify in real time in vascular conduits using currently available techniques. There are 2 major findings of this study. First, OCT is an accurate and reproducible way to evaluate conduits for pathology that would be otherwise undetectable by means of clinical evaluation. Second, traumatic and pre-existing pathology in bypass conduits is extremely common, occurring to some degree in almost every evaluated conduit.

Intraoperative OCT imaging might also provide insight into pathophysiologic events after grafting. In segments of SVGs, the ex vivo measurement of IMT ratio by using OCT directly correlated with the levels of MMP-2 in that same specimen. MMP-2 has been shown to be a key contributor to the pathogenesis of neointimal hyperplasia in experimental models. ${ }^{15}$ Others report that pre-existing intimal thickening in vein specimens correlates with accelerated neointimal hyperplasia in a vein culture model ${ }^{16}$ and predicts failure of vascular surgery bypass grafts. ${ }^{17,18}$ We suggest that pre-existing intimal disease in the RA might also influence the function of this conduit after CABG. IMT ratio in an RA segment was found to inversely correlate with production of the vasodilators prostacyclin and nitric oxide. Others have shown that smooth muscle cells from atherosclerotic RAs have a more depolarized membrane potential relative to smooth muscle cells from control specimens. ${ }^{19}$ In combination, these abnormalities in the atherosclerotic RA might represent a phenotype prone to spasm. If confirmed,
OCT might provide an objective strategy for selecting conduits or portions of conduits at reduced risk for accelerated neointimal hyperplasia or postoperative spasm.

In addition to in situ screening before harvest, ex vivo OCT imaging might serve as a valuable quality assurance tool that gives real-time feedback about harvesting technique. On the basis of success with endoscopic vein harvest, several centers have initiated ERAH. ${ }^{20,21} \mathrm{Al}$ though endoscopic harvest inevitably requires more direct conduit manipulation than an open approach, there is no evidence that this has affected SVG patency. ${ }^{22}$ Preliminary reports of ERAH also suggest good early clinical outcomes. ${ }^{20,21}$ However, angiographic confirmation of graft patency has been limited in most of these reports to patients with symptoms, raising concerns about detection and attrition biases. ${ }^{23}$ It is widely believed that the RA graft is more susceptible to procurement-related trauma than the SVG. Our analysis corroborates this belief by showing more intimal disruption in RA grafts after ERAH when compared with either open harvested RA grafts or endoscopically harvested SVGs. Of note, nearly all the intimal abnormalities were linked to plausible explanations, such as excessive tension at branch points or crush injury to the RA adjacent to the balloon of the trochar port. These data provide avenues for further improvement in ERAH in our practice.

Ex vivo OCT imaging also documented the presence of residual clot strands within endoscopically harvested conduits. Blood flow in the conduit is stopped before the 
administration of heparin caused by $\mathrm{CO}_{2}$ insufflation at greater than venous pressure during endoscopic vein harvest and use of an upper arm tourniquet during ERAH. Fibrin formation under these circumstances does not seem surprising. In addition, the presence of platelets and thrombin raises concern that these strands could serve as a nidus for further thrombus formation in the postoperative period. Prior reports describe the use of a systemic heparin bolus (eg, $5000 \mathrm{U}$ ) before the onset of these procedures. However, the efficacy of this approach has not been established. OCT imaging provides an objective end point for optimizing heparin dosing and balancing the risks of heparin-related bleeding versus thrombus formation.

Our study has several limitations. Only distal RA graft and SVG segments were available for analysis. Distal segments of RA grafts have been reported to contain more intrinsic histopathology, ${ }^{24}$ and discarded segments are likely handled with less attention to intimal integrity than the portion of the conduit used for the bypass. Hence the incidence of histopathology might have been artificially heightened. The only way to fully appreciate heterogeneous changes is to analyze the entire conduit. A second issue is the lack of an SVG control group harvested by using an open method; ongoing studies are underway to address both of these limitations. Although we believe that OCT provides an evaluation of bypass conduits that has not been previously possible, direct comparison of OCT with clinically available modalities, such as intravascular ultrasonography and high-resolution computed tomographic scanning of the forearm, was not performed. Nonetheless, the imaging resolutions that are reported for these other techniques are clearly insufficient for detecting the subtle intimal defects shown in this report. ${ }^{25-28}$

In conclusion, we established OCT as a clinically relevant method with the resolution, accuracy, and reliability to detect abnormalities in conduits used for CABG. Pre-existing and traumatically induced abnormalities were more common than might be otherwise expected in conduits used for bypass grafting. These pathologies are likely to influence the development of neointimal hyperplasia in SVGs and spasm in the RA graft and the risk of acute thrombosis in either graft. The simplicity of OCT scanning for vascular pathology suggests that OCT-based decision making for conduit selection is safe and feasible. Therefore an appropriately powered trial to establish the correlation with patient outcome is clinically justifiable. ${ }^{11,25}$

\section{References}

1. Campeau L, Enjalbert M, Lesperance J, Bourassa MG. Course of angina 1 to 12 years after aortocoronary bypass surgery related to changes in grafts and native coronary arteries. Can J Surg. 1985;28: 496-8.

2. Goldman S, Zadina K, Moritz T, et al. Long-term patency of saphenous vein and left internal mammary artery grafts after coronary artery bypass surgery: results from a Department of Veterans Affairs Cooperative Study. J Am Coll Cardiol. 2004;44:2149-56.

3. Mack MJ, Osborne JA, Shennib H. Arterial graft patency in coronary artery bypass grafting: what do we really know? Ann Thorac Surg. 1998;66:1055-9.

4. Fitzgibbon GM, Kafka HP, Leach AJ, et al. Coronary bypass graft fate and patient outcome: angiographic follow-up of 5,065 grafts related to survival and reoperation in 1,388 patients during 25 years. J Am Coll Cardiol. 1996;28:616-26.

5. Wagner R. Intimal protection of bypass-veins during intraoperative storage in blood or Euro-Collins-solution: the role of medium, temperature, and time. Thorac Cardiovasc Surg. 1990;38:151-6.

6. Thatte HS, Biswas KS, Najjar SF, et al. Multi-photon microscopic evaluation of saphenous vein endothelium and its preservation with a new solution, GALA. Ann Thorac Surg. 2003;75:1145-52.

7. Souza DS, Bomfim V, Skoglund H, et al. High early patency of saphenous vein graft for coronary artery bypass harvested with surrounding tissue. Ann Thorac Surg. 2001;71:797-800.

8. Thatte HS, Khuri SF. The coronary artery bypass conduit: I. Intraoperative endothelial injury and its implication on graft patency. Ann Thorac Surg. 2001;72(suppl):S2245-52.

9. Stamper D, Weissman NJ, Brezinski M. Plaque characterization with optical coherence tomography. J Am Coll Cardiol. 2006;47:C69-79.

10. Gerckens U, Buellesfeld L, McNamara E, Grube E. Optical Coherence Tomography (OCT). Potential of a new high-resolution intracoronary imaging technique. Herz. 2003;28:496-500.

11. Jesuthasan LS, Angus JA, Rosenfeldt FL. In vitro comparison of glyceryl trinitrate-verapamil with other dilators of human saphenous vein. ANZ J Surg. 2003;73:313-20.

12. Meissner OA, Rieber J, Babaryka G, et al. Intravascular optical coherence tomography: comparison with histopathology in atherosclerotic peripheral artery specimens. J Vasc Interv Radiol. 2006;17:343-9.

13. Mintz GS, Popma JJ, Pichard AD, et al. Patterns of calcification in coronary artery disease. A statistical analysis of intravascular ultrasound and coronary angiography in 1155 lesions. Circulation. 1995; 91:1959-65.

14. Manchio JV, Gu J, Romar L, et al. Disruption of graft endothelium correlates with early failure after off-pump coronary artery bypass surgery. Ann Thorac Surg. 2005;79:1991-8.

15. Corpataux JM, Naik J, Porter KE, London NJ. A comparison of six statins on the development of intimal hyperplasia in a human vein culture model. Eur J Vasc Endovasc Surg. 2005;29:177-81.

16. Wilson YG, Davies AH, Southgate K, et al. Vein quality influences neointimal hyperplasia in an organ culture model of human saphenous vein. Eur J Vasc Endovasc Surg. 1997;13:557-62.

17. Beattie DK, Sian M, Greenhalgh RM, Davies AH. Influence of systemic factors on pre-existing intimal hyperplasia and their effect on the outcome of infrainguinal arterial reconstruction with vein. Br J Surg. 1999;86:1441-7.

18. Panetta TF, Marin ML, Veith FJ, et al. Unsuspected preexisting saphenous vein disease: an unrecognized cause of vein bypass failure. $J$ Vasc Surg. 1992;15:102-10.

19. Zhang Y, Janssen L, Chu FV. Atherosclerosis of radial arterial graft may increase the potential of vessel spasm in coronary bypass surgery. J Thorac Cardiovasc Surg. 2005;130:1477-8.

20. Sato M, Suenaga E, Senaha S, Furutachi A, Morokumia H. Initial experience in endoscopic radial artery harvesting. Kyobu Geka. 2006; 59:123-5.

21. Chiu KM, Li SJ, Chen JS, Lin TY, Chan CY, Chu SH. Endoscopic radial artery harvest for coronary artery bypass surgery. J Formos Med Assoc. 2006;105:384-9.

22. Yun KL, Wu Y, Aharonian V, et al. Randomized trial of endoscopic versus open vein harvest for coronary artery bypass grafting: sixmonth patency rates. J Thorac Cardiovasc Surg. 2005;129:496-503.

23. Juni P, Altman DG, Egger M. Systematic reviews in health care: assessing the quality of controlled clinical trials. BMJ. 2001;323:42-6.

24. Chowdhury UK, Airan B, Mishra PK, et al. Histopathology and morphometry of radial artery conduits: basic study and clinical application. Ann Thorac Surg. 2004;78:1614-21. 
25. Kume T, Akasaka T, Kawamoto T, et al. Assessment of coronary intima-media thickness by optical coherence tomography: comparison with intravascular ultrasound. Circ J. 2005;69:903-7.

26. Kume T, Akasaka T, Kawamoto T, et al. Assessment of coronary arterial plaque by optical coherence tomography. Am J Cardiol. 2006; 97:1172-5.

27. Meissner OA, Rieber J, Babaryka G, et al. Intravascular optical coherence tomography: differentiation of atherosclerotic plaques and quantification of vessel dimensions in crural arterial specimens. Rofo Fortschr Geb Rontgenstr Neuen Bildgeb Verfahr. 2006;178: 214-20.

28. Leber AW, Knez A, von Ziegler F, et al. Quantification of obstructive and nonobstructive coronary lesions by 64-slice computed tomography: a comparative study with quantitative coronary angiography and intravascular ultrasound. J Am Coll Cardiol. 2005; 46:147-54.

\section{Discussion}

Dr Sahar Gideon (Beer Sheba, Israel). I would like to know whether you could determine any correlation between intimal hyperplasia and risk factors of these patients?

Dr Burris. We did not look in this particular study at the risk factors for intimal hyperplasia. We analyzed discarded segments of these conduits that were given to us after bypass surgery. The primary thrust of this study was really to validate OCT's accuracy and reproducibility as an evaluation tool. However, those are definitely questions that we are interested in pursuing and will be pursuing in the future. We are interested in the pathological significance of these preexisting pathologies and how they relate to patency. I think we have demonstrated that OCT has the ability to detect these pathologies, which will be the first step in understanding their physiological importance.

Dr Gideon. Do you have any information about the ITA?

Dr Burris. Yes. Excellent question. Thank you. It is well known that the ITA is the best conduit for bypass surgery. It is typically harder to get a discarded segment to scan, but on several occasions, we have observed 4 discarded segments of distal ITA, and in none of those samples have we noticed any type of plaque, intimal thickening, or any other pathology that is detectable with OCT. These findings suggest the relative health of ITAs and also corroborate our histologic findings that there is excellent endothelial integrity and low disease in the ITA.

Dr Hakob Davtyan (San Bernardino, Calif). I noticed these little strands of clots in the endoscopically harvested veins in my practice, and at this point, I stopped doing endoscopic harvest until we can figure out how we are going to deal with this. In your findings, did it change the way you harvest the vein or just the way you practice coronary bypass surgery with saphenous veins?

Dr Burris. Thank you for your excellent question. We did notice the presence of these clot strands associated with the endoscopic harvest in RAs and saphenous veins. Thus far, we have only looked at distal discarded segments of these vessels, and therefore we open ourselves to some sampling error because the distal portions of these segments are known to have more pre-existent pathology. It seems reasonable to hypothesize that there would also be clot strands in the portion of the conduit that is being grafted. However, that is the next step for us. We will be doing these scans in situ, in an operative setting with sterile probes to determine the heterogeneity and the prevalence of these pathologies. We will be looking at plaque as well in the more proximal portions of these grafts, which will be grafted. After we complete that study and we understand the pathology a little bit more thoroughly, we will consider revising our protocol. Some have hypothesized that heparinization before endoscopic harvest of the saphenous vein could alleviate that, and we will be using OCT to investigate that as a possible solution. I think OCT is a very good modality to really have a strong end point to objectively evaluate that problem.

Dr Mustafa Cikirikcioglu (Geneva, Switzerland). How long was the mean length of the segments that you evaluated?

Dr Burris. The mean length that we evaluated was about $4.5 \mathrm{~cm}$ in veins, and typically, it was a little bit shorter in RAs, about $3 \mathrm{~cm}$, and on the rare occasion that we did get an ITA, it was generally less than a centimeter in length. We only observed 4 of those, though.

Dr Cikirikcioglu. I do not know whether it is soft or rigid, but if you extend the length of the evaluated segment, what do you think about creating new lesions by your device?

Dr Burris. I am sorry, excuse me?

Dr Cikirikcioglu. You evaluated very short segments, but what do you think about evaluating longer segments with your device? Do you think you can create new lesions with your device if you will evaluate longer segments?

Dr Weisel. To ask the question another way, the plan here in the future is to evaluate the entire graft before putting it in. Are you going to injure the graft trying to evaluate it?

Dr Burris. Thank you for the question. I am sorry, I did not understand. That was a concern, of course, when we first started this study. We did a cadaver model, actually, at our institution, where we used 12 cadavers, and we harvested the RA bilaterally using an endoscopic technique. We performed serial scans in which we performed an initial scan during which we passed the probe into the RA. We then retracted it and performed another scan just to evaluate whether there was any intimal injury induced by the insertion of the probe itself, and we did not notice any detectable intimal injury because of just the insertion of the OCT probe alone.

Dr Paul Kurlansky (Miami, Fla). Just a quick question about the technology. Is this a technology that will help us visualize things in a blood-filled field as a potential alternative or component to echocardiography just as an imaging technique?

Dr Burris. Excellent question. For anyone who has heard about OCT's application in coronary arteries, where it is currently underway in US Food and Drug Administration trials, they might know that the one limitation of OCT is that it does not image through blood, unlike intravascular ultrasonography. In our particular application, that is an easily surmountable problem. We exsanguinate the arm with a tourniquet before doing these scans and inject a small amount of saline, less than $20 \mathrm{~mL}$, to clear the blood from both the vein and the RA, and this provides us with a bloodless conduit to evaluate with OCT. But that is definitely something that we have considered. It is one of the advantages of this application over the coronary application, where you have to inflate an occlusion balloon in the coronary arteries during percutaneous coronary intervention. Therefore I think we have a very minimally invasive and simple solution to that problem in our application, and it has not proved to be a significant factor.

Dr John D. Puskas (Atlanta, Ga). Nicholas, one last question. There is a wealth of data in peer-reviewed publications demonstrating that the short- and intermediate-term patency of grafts harvested by means of endoscopic techniques does not differ from the patency of grafts harvested by using the older open technique. Your data suggested that ought not to be the case. Can you explain this discrepancy?

Dr Burris. Thank you for the question. It certainly has been documented that the patency rate of endoscopic veins is com- 
parable with that of open-harvested veins. In this particular study, because we did not look at the patency of the evaluated conduits, we are limited in how we can comment on that discrepancy. I defiantly think that OCT has the ability to detect the important pathologies that have been suspected in this midterm to late-term failure.
Most recently, there was the PREVENT IV trial, which some people are familiar with, in which $80 \%$ of these veins were harvested endoscopically and there was an angiographic 1-year failure rate of $40 \%$ for saphenous veins. This is an example of some recent research that shows there might still be a problem with endoscopically harvested saphenous veins. 